\title{
Resistive Cerebral Blood Flow as a Potential Marker of Subclinical Brain Damage in Essential Hypertension
}

\author{
Sergio González-García ${ }^{1}$, Zenaida Hernández-Díaz¹, Luis Quevedo-Sotolongo², \\ Marisol Peña-Sánchez ${ }^{1}$, Yaima Pino-Peña1, Rebeca Fernández-Carriera ${ }^{1}$, \\ Caridad Menéndez-Saínz ${ }^{1}$, Alina González-Quevedo', \\ Janis Eells ${ }^{3}$ \\ ${ }^{1}$ Institute of Neurology and Neurosurgery, La Habana, Cuba \\ ${ }^{2}$ Cira García Central Clinic, La Habana, Cuba \\ ${ }^{3}$ College of Health Sciences, University of Wisconsin-Milwaukee, Milwaukee, USA \\ Email: sergiogg@infomed.sld.cu
}

Received 3 March 2014; revised 6 April 2014; accepted 15 April 2014

Copyright (C) 2014 by authors and Scientific Research Publishing Inc.

This work is licensed under the Creative Commons Attribution International License (CC BY). http://creativecommons.org/licenses/by/4.0/

(c) (i) Open Access

\section{Abstract}

Introduction: Subclinical brain damage in essential hypertension is more prevalent than cardiovascular or renal impairment; nevertheless, screening for nervous system involvement is difficult due to the low accessibility and high costs of these techniques. Objective: To assess the frequency of silent target organ damage in a cohort of asymptomatic hypertensive patients and to evaluate the potential usefulness of carotid ultrasonographic (US) variables as predictors of subclinical brain damage. Patients and Methods: Thirty four neurologically asymptomatic subjects (mean age 59 years) with essential hypertension were included. Target organ damage was evaluated: degree of hypertensive retinopathy, heart, kidney and brain. Structural and hemodynamical carotid Doppler US parameters were also investigated. Results: The brain was the most frequently affected target organ (70.6\%), followed by the heart (67.9\%) and kidney $(58.6 \%)$. Carotid US parameters showed no association of intima media thickness with brain MRI results; nevertheless, decreased diastolic flow velocity and increased resistive index pointed to a resistive carotid flow pattern in patients with classical brain MRI lesions and predicted subclinical lesions with a sensitivity of $70 \%$ and $74 \%$ and a specificity of $72 \%$ and $80 \%$ respectively. Conclusions: This study supports previous findings that place the brain as the most frequently affected target organ in essential hypertensive patients and sheds more light on the potential usefulness of carotid structure and hemodynamics as imaging biomarkers of subclinical brain lesions. 


\section{Keywords}

\section{Subclinical Brain Damage, Essential Hypertension, Carotid Doppler}

\section{Introduction}

Hypertension (HT) is the most common health condition affecting adult individuals in populations throughout the world. Currently a billion persons have hypertension [1]-[3]. It represents in itself a disease, as well as a risk factor for other diseases. Chronic hypertension affects vascular systems of various organs, especially the heart, brain, kidney and retina [2] [3]. The brain is one of the target organs which is frequently affected by high blood pressure (HBP), and constitutes the major modifiable risk factor for ischemic and hemorrhagic stroke, as well as small-vessel disease. It has been reported to predispose the development of white matter hyperintensities (WMH), lacunar infarction and microbleedings which are mostly silent, and has been linked to the occurrence of vascular dementia and other malfunctions of the central nervous system [1] [2] [4] [5]. Currently, HT guidelines recognize the heart and kidneys as the main target organs affected by high blood pressure [3]. However, Henskens et al. showed that silent cerebrovascular damage is more common in hypertensive subjects than cardiorrenal impairment [6]. Likewise, the European Guidelines for the Management of Hypertension reported that silent brain lesions were more frequent than subclinical cardiac and renal damage [2]. The evaluation of the consequences of HT on the heart and kidneys has become a routine clinical practice for years. However, methods for demonstrating asymptomatic neurological damage by HT are still sought, as there are no accessible and cost effective evaluation techniques to assess the damage.

The suggested underlying mechanism linking elevated blood pressure levels with WMH includes hypertension-induced structural vessel changes in microcirculation such as lipohyalinosis and fibrinoid degeneration [7]. Also high blood flow affecting macrocirculation causes arterial remodeling and atheromatosis [8]. Changes in macrocirculation can be assessed by high-resolution B-mode ultrasonography. Limited studies have evaluated the relation between WMHs on brain MRI and ultrasound assessments of structural macrocirculation-vessel changes with partly conflicting findings [9]-[12]. Due to the high cost and low availability of MRI studies, the use of carotid Doppler as a potential marker of asymptomatic cerebral damage remains a very attractive option. The aim of this investigation was to assess the frequency of silent target organ damage in a cohort of asymptomatic hypertensive patients and to evaluate the potential usefulness of carotid ultrasonographic variables as predictors of target organ damage.

\section{Patients and Methods}

Hypertensive patients were referred by physicians to the Neurology and Neurosurgery Institute from January 2012 to June 2013 for evaluation of the degree of retinopathy. Confirmation of the status of each patient was performed by a neurologist and an ophthalmologist, considering the Seventh Report on Prevention, Detection, Evaluation, and Treatment of HTA [13]. Systolic and diastolic blood pressure (SBP and DBP respectively) were measured in all subjects in the right arm with an aneroid sphygmomanometer, in a sitting position and after a resting period of 5 minutes. They underwent an ophthalmological examination to establish signs of hypertensive retinopathy, classified according to Scheie [14]. Inclusion criteria were: men and women aged 40 to 75 years with a history of essential hypertension, and without neurological brain disease. Exclusion criteria were: secondary causes of HT, carotid stenosis $>70 \%$, previous cerebrovascular disease or other neurological diseases.

All hypertensive patients collected a urine sample for semiquantitative determination of microalbuminuria ( $\mu \mathrm{alb}$ ), employing the Microalb-Látex (HELFA Diagnostics) method. The presence of $\mu$ alb was established when concentrations $>0.02 \mathrm{~g} / \mathrm{L}$ were detected. The Cockroft-Gault formula was employed to estimate creatinine clearance [15] and glomerular filtration rates (DGFR) were considered decreased when values $<70 \mathrm{~mL} / \mathrm{min}$ were encountered.

Brain MRI study was performed with a Siemens (MAGNETOM Concerto) 0.2 Tesla open permanent magnet scanner. All scans were controlled and stored in the image analysis center of the Central Clinic "Cira Garcia" in Havana. Brain MRI changes-white matter hyperintensities (WMH), lacunar infarcts (LI) and dilated VirchowRobin spaces ( $>2 \mathrm{~mm}$ ) were classified in increasing severity as detailed in a previous work [16]. Cerebral atro- 
phy (Yes or No) was also evaluated. The lesions found on MRI were also classified as classical or non-classical. Lesions were considered classical when lacunar infarcts and/or white matter hyperintensities were present; and non-classical when there were only dilated Virchow-Robin spaces (DVRS) and/or brain atrophy (A). All MRI scans were evaluated by the same investigator (LQS) who was blinded to the ultrasound parameters and clinical variables of the study population.

The echocardiographic study was performed with a conventional M-mode echocardiograph (PROSOUND ALOKA Alpha model 10). Thickness of the interventricular septum (IVS) and the rear wall (RW) were measured. Reference values were: IVS $<11 \mathrm{~mm}$ and RW $<7 \mathrm{~mm}-11 \mathrm{~mm}$. Diastolic dysfunction was recorded as Yes or No.

The presence of target organ damage (Yes or No) was evaluated as follows: Brain: Presence of classic lesions (LI and/or WMH) on brain MRI. Heart: Presence of diastolic dysfunction and/or IVS and/or RW hypertrophy by echocardiography. Kidney: Presence of $\mu$ alb and/or decreased glomerular filtration rate.

Neurosonology Protocol. An experienced neurosonology investigator (ZHD), blinded to clinical and MRI data, performed the ultrasound investigations within one month after MRI. Carotid ultrasound scanning was performed using an ALOKA brand PROSOUND alpha model 10 scanner equipped with a 7.5 - $13 \mathrm{MHz}$ linear transducer, placed on each side of the patient's neck, after application of gel transmitter. The scan was performed in a room with the necessary hygienic conditions and dim light for adequate appreciation of details. The patient was placed supine on a stretcher; his neck slightly extended and rotated with both arms close to the body, in order to obtain maximum descent of the shoulders. The same scanning sequence of the carotid axis was always followed to avoid confusion and mistakes. The following indicators were measured:

- Intima-Media thickness (IMT): Measurements were made bilaterally at the level of the common carotid artery (CCA), $1 \mathrm{~cm}$ from the bulb at the end of diastole.

- Peak systolic and diastolic velocities and mean velocity of flow at the internal carotid artery (PSV, PDV and MVF respectively).

- $\quad$ Resistance and pulsatility indexes (RI and PI respectively).

\subsection{Statistical Analyses}

Frequencies of demographic and clinical variables were calculated and continuous variables were tested for normal distribution using Kolmogorov-Smirnov test. Associations between categories of variables were measured by the $\chi^{2}$ test. Differences between groups for continuous variables were assayed employing the MannWhitney U test or Kruskall-Wallis tests. Statistical calculations were performed with Statistica 8.0 for Windows (StatsoftInc, 2010). Statistical significance was achieved if $p<0.05$ (two-sided $p$ values).

\subsection{Ethical Procedures}

The research was approved by the Ethics Committee of the Neurology and Neurosurgery Institute, and conducted according to the provisions of the latest version of the Declaration of Helsinki. The purposes of the research were explained to all patients who agreed to participate in the study and signed informed consent.

\section{Results}

\subsection{Characteristics of Hypertensive Patients and Target Organ Damage by Hypertension}

The mean age of the 34 patients was 59 years (40 - 70 years); 22 were female (64.7\%), 22 (64.7\%) were of white ethnic origin and 12 (35.8\%) were black ethnic or mixed. Means $( \pm$ SD) for SBP and DBP were $141.8 \pm$ $17.9 \mathrm{mmHg}$ and $93.5 \pm 11.7 \mathrm{mmHg}$ respectively. The duration of hypertension (mean \pm SD) was $15.4 \pm 10.4$ years (2 - 50 years); 33\% suffered from hypertension for less than 10 years and $94.1 \%$ were subjected to some scheme of antihypertensive medication. The classification of patients according to BP and grade of retinopathy are shown in Table 1. An association between BP group and grade of retinopathy was observed $\left(\chi^{2}=21.8 ; p=\right.$ 0.0092).

The results of brain MRI studies are summarized in Table 2. Hyperintensities were found in 23 patients (67.6\%), whereas 11 subjects showed no hyperintense lesions. Grade I hyperintensities were observed in 8 patients, grade II in 10 and only 5 were classified as grade III. The presence of lacunar infarctions, dilated Virchow-Robin spaces and atrophy were detected in 6 (17.6\%), 17 (50.0\%) and 16 (47.1\%) patients respectively. 
Table 1. Classification of patients according to blood pressure and grade of retinopathy.

\begin{tabular}{ccc}
\hline Blood pressure (mmHg) & $\mathbf{n}$ & $\mathbf{\%}$ \\
\hline$<120 / 80$ & 4 & 11.8 \\
$120-139 / 80-89$ & 15 & 44.1 \\
$140-159 / 90-99$ & 7 & 20.6 \\
$\geq 160 / 100$ & 8 & 23.5 \\
Grade of retinopathy & $\mathbf{n}$ & $\mathbf{\%}$ \\
0 & 2 & 6.1 \\
I & 14 & 42.4 \\
II & 14 & 42.4 \\
III & 3 & 9.1 \\
\hline
\end{tabular}

\begin{tabular}{|c|c|c|c|c|}
\hline \multicolumn{2}{|c|}{ Brain MRI $(n=34)$} & \multirow{2}{*}{$\begin{array}{c}\mathrm{n} \\
11\end{array}$} & \multirow{2}{*}{$\begin{array}{c}\% \\
32.4\end{array}$} & \multirow{2}{*}{ Total } \\
\hline \multirow{4}{*}{$\begin{array}{l}\text { White matter } \\
\text { hyperintensities }\end{array}$} & No & & & \\
\hline & Grade I & 8 & 23.5 & \multirow{4}{*}{$23(67.6 \%)$} \\
\hline & Grade II & 10 & 29.4 & \\
\hline & Grade III & 5 & 14.7 & \\
\hline \multirow{3}{*}{ Lacunar Infarctions } & No & 28 & 82.4 & \\
\hline & $\leq 3$ & 6 & 17.6 & \multirow{2}{*}{$6(17.6 \%)$} \\
\hline & $>3$ & 0 & - & \\
\hline \multirow{3}{*}{$\begin{array}{l}\text { Dilated Virchow Robin } \\
\text { spaces }\end{array}$} & No & 17 & 50.0 & \multirow{4}{*}{$17(50.0 \%)$} \\
\hline & $\leq 3$ & 4 & 11.8 & \\
\hline & $>3$ & 13 & 38.2 & \\
\hline \multirow{2}{*}{ Atrophy } & No & 16 & 47.1 & \\
\hline & Yes & 16 & 47.1 & $16(47.1 \%)$ \\
\hline
\end{tabular}

The final classification of patients according to brain MRI findings showed that only 4 patients $(11.8 \%)$ were free of lesions, 6 (17.6\%) classified as non-classical and 24 patients (70.6\%) had classical lesions.

Patients with no lesions and with non-classical lesions were grouped and compared to patients with classical lesions according to duration of hypertension in years and SBP respectively (Figure 1). It can be observed that the presence of classical lesions on MRI was associated with a longer duration of hypertension and higher values of SBP.

Table 3 shows the effect of essential HT on the different vascular beds in patients enrolled in the study. Heart and kidney were assessed in 28 and 29 hypertensive patients respectively, of whom $67.9 \%$ had some type of damage to the heart and $58.6 \%$ had some type of renal damage. Classical brain lesions were the most frequent (70.6\%), thus establishing that brain was the most frequently damaged organ in the group of hypertensive patients studied.

The complete assessment of the 3 systems (heart, kidney and brain) was achieved in 27 hypertensive subjects. Figure 2 shows the percentage of patients according to the affected target organs as a consequence of hypertension. Damage to the 3 vascular beds was found in $31 \%$ of all hypertensive subjects; the combinations brain-heart and brain-kidney followed in frequency (23.1\% each), and $11.5 \%$ of the subjects had brain damage without cardiorrenal impairment. Of the 22 subjects with cardiorrenal damage, 91\% showed some brain impairment, while 4 patients without cardiorrenal impairment already had brain lesions.

\subsection{Carotid Doppler Findings and Target Organ Damage}

Classical lesions (LI and/or WMH) on brain MRI were associated with a more resistive carotid flow pattern, ie increased vascular resistance index (RI) and decreased peak diastolic velocity (PDV) (Figure 3). Pulsatility in- 
dex, peak systolic velocity and intimal thickening were not related to brain injuries (data not shown).

Heart and kidney damage were not associated with any of the Doppler ultrasound parameters explored.

As resistance index and peak diastolic velocity were associated with the presence of classical lesions, ROC curves were calculated (Figure 4). ROC analysis showed that a cutoff value of 0.61 in RI predicted the appearance of classical lesions (WMH and/or LI) with a sensitivity of $74 \%$ and a specificity of $80 \%$. On the other hand, PDV values below 20.6 predicted classical lesions with a sensitivity and specificity of $70 \%$ and $72 \%$ respectively.

Table 3. Target organ damage in hypertensive subjects.

\begin{tabular}{ccc}
\hline Variables of target organ damage & $\mathrm{n}$ & $\%$ \\
\hline Echocardiogram (n = 28) & 9 & 36.0 \\
No alterations & 7 & 25.0 \\
Diastolic dysfunction (DD) & 0 & 0 \\
IVS and/or RW hypertrophy & 12 & 42.9 \\
DD + IVS and/or RW hypertrophy & & \\
Kidney function (n = 29) & 12 & 41.4 \\
No alterations & 7 & 24.1 \\
$\mu$ alb & 5 & 17.2 \\
DGFR & 5 & 17.2 \\
$\mu$ alb + DGFR & & \\
MRI (n = 34) & 4 & 11.8 \\
No alterations & 6 & 17.6 \\
Non classical lesions & 24 & 70.6 \\
Classical lesions &
\end{tabular}

IVS—Interventricular Septum; RW—Rear Wall; DGFR—Glomerular Filtration Rates; $\mu$ alb - Microal-Buminuria.
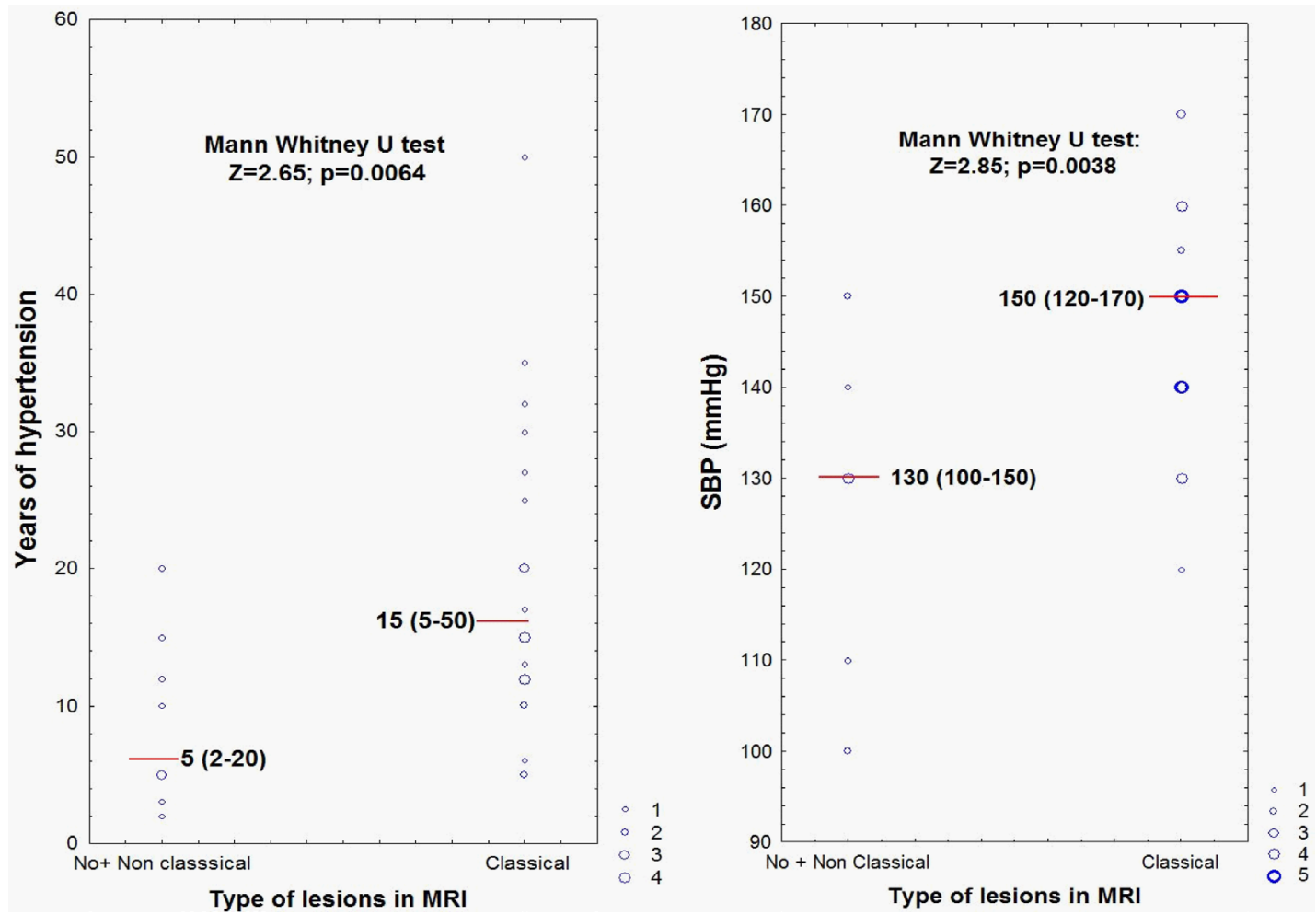

Figure 1. MRI findings according to years of hypertension and SBP values. 


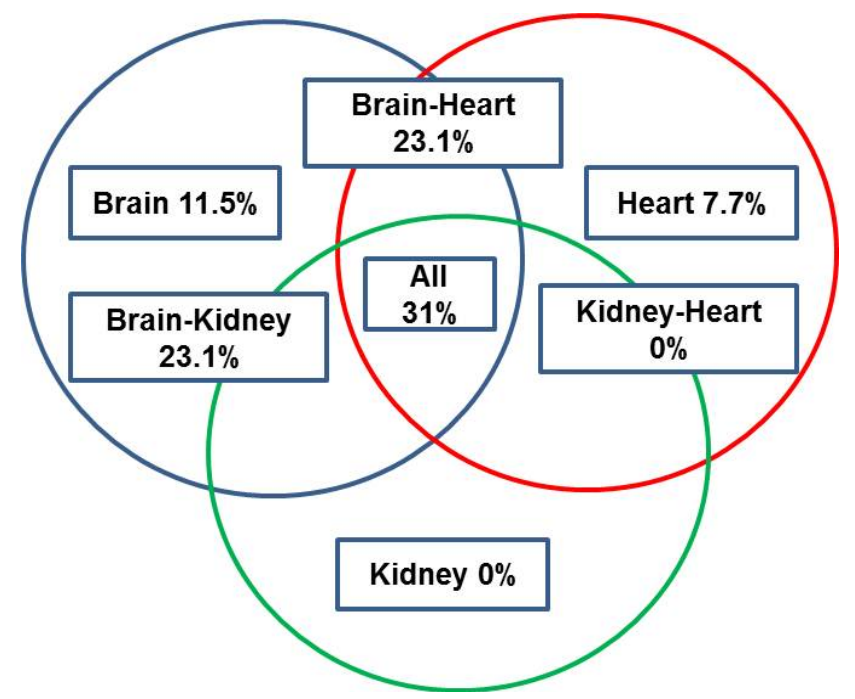

Figure 2. The complete evaluation of the systems affected by HT.
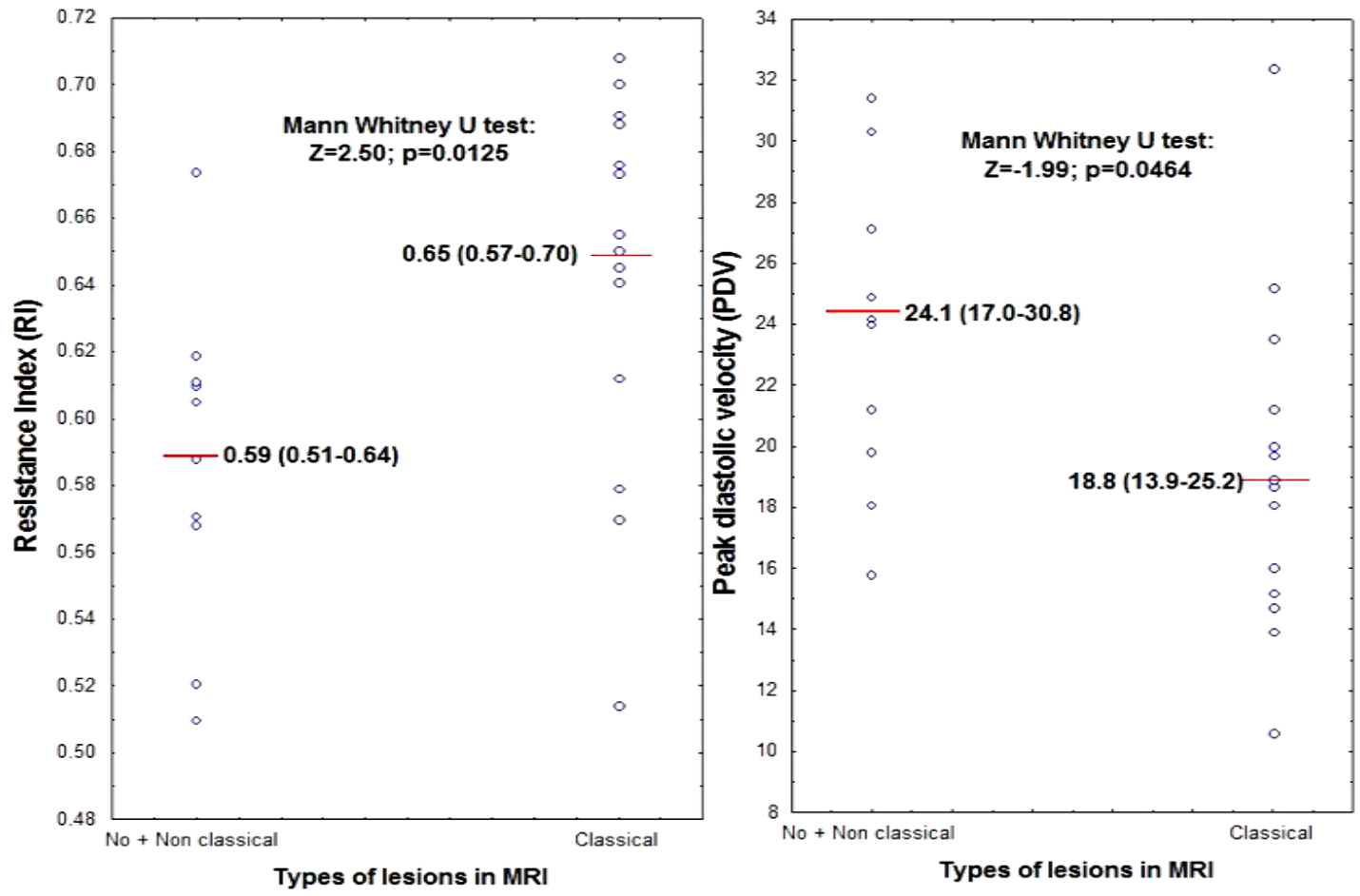

Figure 3. RI and PDV according to the brain lesions in MRI.

\section{Discussion}

The present cross-sectional study in a cohort of 34 hypertensive patients (mean age 59 years) showed that the most frequently affected target organ was the brain, as $70.6 \%$ had classical brain MRI lesions (associated with the years of hypertension and SBP); while cardiac and renal involvement was seen in $67.9 \%$ and $58.6 \%$ respectively. Damage to the three target organs was found in 31\% of patients and of the 22 subjects with cardio-renal involvement, 91\% had classical brain MRI lesions. Carotid ultrasound parameters and cardiorrenal variables explored were not associated; nevertheless, decreased peak diastolic velocity and increased resistive index predicted classic brain MRI lesions with a sensitivity of $70 \%$ and $74 \%$ and a specificity of $72 \%$ and $80 \%$ respectively. 


\section{ROC Curve IR}

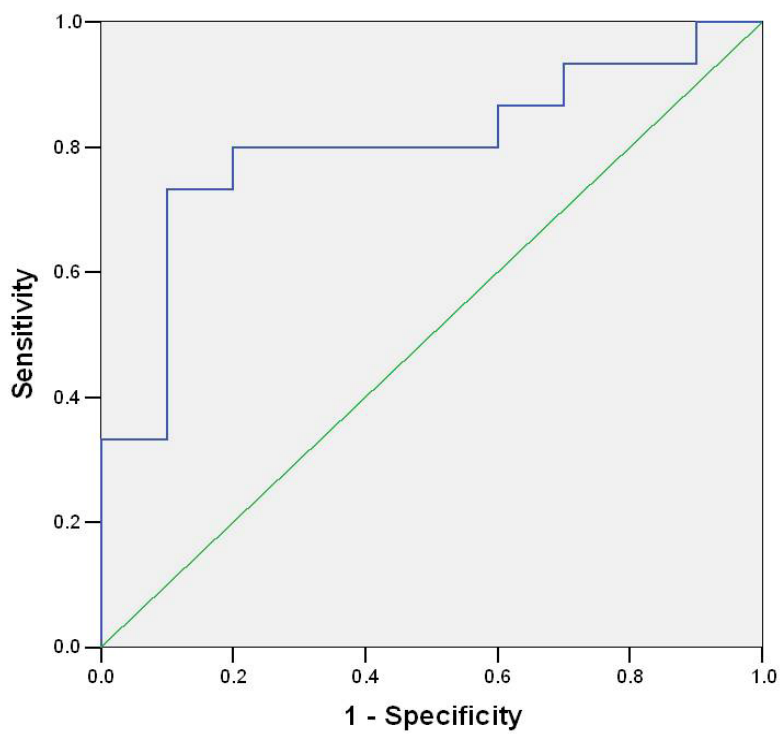

\begin{tabular}{ccc}
\hline Ultrasound variable & Cut off & Sensitivity \\
\hline RI & 0.61 & 0.74 \\
DVF & 20.6 & 0.70
\end{tabular}

ROC Curve DVF

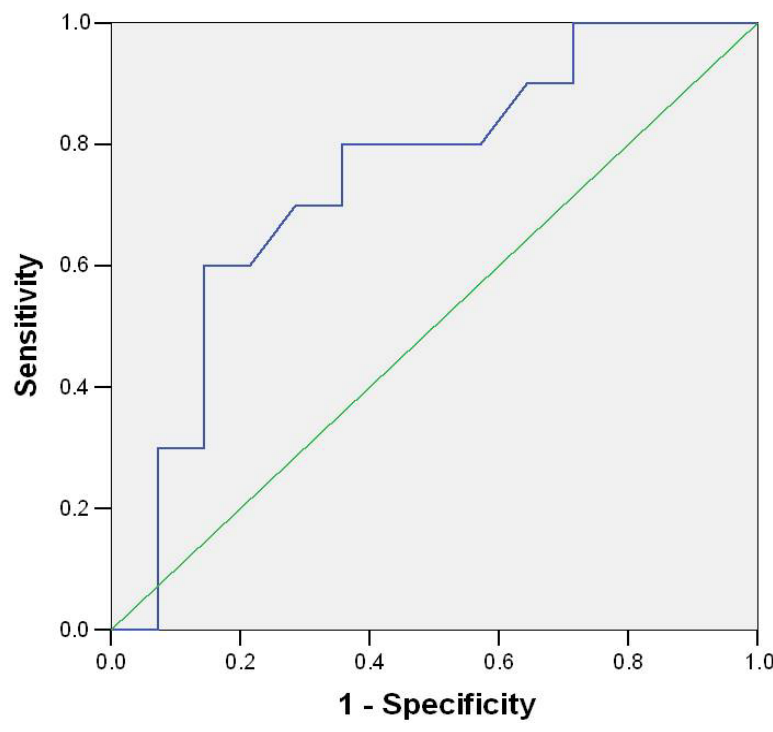

\begin{tabular}{cc} 
Specificity & Area and $95 \%$ Confidence Interval \\
0.80 & $0.800(0.619-0.981)$ \\
0.72 & $0.743(0.538-0.947)$ \\
\hline
\end{tabular}

Figure 4. ROC for RI and PDV.

Other authors have reported the brain as the most frequently affected organ, when compared with the heart and kidney [17]-[19]. While the entire vasculature is exposed to the action of HT, the particular effects on small vessels is crucial in the severity of damage to cerebral structures [20] [21]. In the ARIC study (Atherosclerosis Risk in Communities Study), conducted in 940 hypertensive subjects aged 55 - 72 years, the prevalence of WMH was 85.0\% [21], while in the Cardiovascular Health Study, conducted in 1452 hypertensive subjects WMH prevalence was 87.0\% [22]. Van Boxtel et al. reported WMH in 65\% of hypertensive patients with a mean age of 57.4 years [23]; while Henskens et al., observed that $44 \%$ of hypertensive patients, with a lower mean age (51.6 years) had brain injuries [6].

Interestingly, in the two cohorts studied in Cuba there was a low percentage of hypertensive patients totally free of lesions in the brain MRI: 26\% in the study of Hernández et al. [18] and 11.8\% in ours. The most striking difference was seen in the distribution of patients according to the presence of classical or non-classical lesions. Hernández [18] detected 38\% and 36\% of patients with classical and non-classical lesions respectively, whereas in the present study a predominance of classical lesions (70.6\%) with respect to non classical (17.6\%) was found. This could be explained by the considerably lower average age of their cohort (44 years) regarding ours.

Considering our results and the aforementioned reports, it can be concluded that the frequency of WMH is high among hypertensive patients, and that it may vary depending on the average age of the study group. The frequency of WMH increases with aging and years of hypertension [24] but it must be taken into account that the way lesions are classified can also influence the results. For example, Henskens et al. [25] classified grade I WMH (according to Fazeka's scale) as non-classical lesions.

From the moment that an individual is exposed to a risk factor to the onset of its clinical consequences there is a period of asymptomatic structural damage. In the case of HT those tests considered "early markers of injury” are actually delayed, as major structural alterations in different organs have been reported as we have previously documented. HT Clinical Guidelines routinely recommend on identifying early damage of the heart or kidney; nevertheless, the routine examination for asymptomatic lesions of the CNS is seldom suggested due to the high cost it implies.

In our study two carotid ultrasound parameters of macrocirculation (PDV and RI) were associated with the extent of structural changes in microcirculation as reflected by WMH burden in a sample of hypertensive indi- 
viduals without prior history of neurological impairment. Both parameters denote early changes in blood flow: increased resistive index and decreased diastolic velocity, indicating a resistive carotid flow pattern in hypertensive patients with classical brain MRI lesions.

There are very few studies correlating structural and hemodynamic carotid US parameters with the extent of brain MRI lesions in otherwise asymptomatic hypertensive patients, and the results are controversial. The most frequently tested variable has been IMT, which was found increased in association to brain MRI lesions [9] [18] [26]. Nevertheless, this association was not encountered very recently by Heliopoulos et al. in a sample of 52 hypertensive patients (mean age 71.4 years), nor in the present study. The lack of association of carotid IMT has been argued as it being a marker of large-vessel rather than small-vessel disease damage [27].

On the other hand, hemodynamic parameters were reported in two studies as not being associated with the severity of brain lesions [26] [27]; although while in the present cohort we found that pulsatility index and diastolic flow velocity measures pointed to a resistive carotid flow pattern in this group of hypertensive patients. Nevertheless, the discrepant results across different studies could be related to sample size. Although the French study was conducted in 198 individuals [9], the rest varied from 34 (in this study) to 52 [18] [26] [27], thus a type II error cannot be ruled out. Other issues to consider are differences in demographic characteristics, specifically considering mean age (44 - 69.3 years) and ethnic origin: Caucasians and Asians [9] [26] [27] and AfroCaucasians in this study and in Hernández et al. [18]. An important concern is related to the criteria employed for patient inclusion, which also varied: all patients with subjective memory complaints [9], all patients were hospitalized and antihypertensive medication was discontinued [26], all patients had WMH in brain MRI, hypertensive patients included from a community study [18]. Finally, different equipment and methodology for structural and hemodynamic measurements also have to be considered.

Our study has two main limitations: First, its cross-sectional design does not allow inferring a cause-effect relationship between brain MRI findings and carotid US variables, and furthermore, the sample size was relatively small, although strict inclusion criteria were applied to avoid including subjects with known underlying diseases which could bias results.

In the present study the carotid US parameters pulsatility index and peak diastolic velocity exhibited relatively good sensitivities and specificities (above 70\%) for the prediction of silent brain lesions in this group of patients. These results establish more grounds in the search of relatively simple, low cost techniques for the prediction of subclinical brain damage in essential hypertension.

\section{Conclusion}

In conclusion, this study supports previous findings that place the brain as the most frequently affected target organ in essential hypertensive patients and sheds more light on the potential usefulness of carotid structure and hemodynamics as imaging biomarkers of subclinical brain lesions. Further observational studies with larger and more homogenous cohorts are needed to determine specifically which extracranial carotid US parameters could be the most adequate ones.

\section{References}

[1] Pérez, C.M.D., Dueñas, H.A., Alfonso, G.J.P., et al. (2006) Guidelines for the Treatment, Prevention and Diagnosis of Arterial Hypertension. Editorial Ciencias Médicas, La Habana.

http://www.bvs.sld.cu/libros texto/hipertension arterial/indice p.htm

[2] Mancia, G., Fagard, R., Narkiewicz, K., Redon, J., Zanchetti, A., Böhm, M., et al. (2013) 2013 ESH/ESC Practice Guidelines for the Management of Arterial Hypertension. Blood Pressure, 23, 3-16.

[3] Shlomai, G., Grassi, G., Grossman, E. and Mancia, G. (2013) Assessment of Target Organ Damage in the Evaluation and Follow-Up of Hypertensive Patients: Where Do We Stand? The Journal of Clinical Hypertension, 15, 742-747.

[4] Al-Sarraf, H. and Philip, L. (2003) Effect of Hypertension on the Integrity of Blood Brain and Blood CSF Barriers, Cerebral Blood Flow and CSF Secretion in the Rat. Brain Research, 975, 179-188. http://dx.doi.org/10.1016/S0006-8993(03)02632-5

[5] Amenta, F., Di Tullio, M.A. and Tomassoni, D. (2003) Arterial Hypertension and Brain Damage-Evidence from Animal Models (Review). Clinical and Experimental Hypertension, 25, 359-380. http://dx.doi.org/10.1081/CEH-120023545

[6] Henskens, L.H., van Oostenbrugge, R.J., Kroon, A.A., Hofman, P.A., Lodder, J. and de Leeuw, P.W. (2009) Detection 
of Silent Cerebrovascular Disease Refines Risk Stratification of Hypertensive Patients. Journal of Hypertension, 27, 846-853. http://dx.doi.org/10.1097/HJH.0b013e3283232c96

[7] Vermeer, S.E., Longstreth, W.T. and Koudstaal, P.J. (2007) Silent Brain Infarcts: A Systematic Review. The Lancet Neurology, 6, 611-619. http://dx.doi.org/10.1016/S1474-4422(07)70170-9

[8] Feihl, F., Liaudet, L. and Waeber, B. (2009) The Macrocirculation and Microcirculation of Hypertension. Current Hypertension Reports, 11, 182-189. http://dx.doi.org/10.1007/s11906-009-0033-6

[9] Kearney-Schwartz, A., Rossignol, P., Bracard, S., et al. (2009) Vascular Structure and Function Is Correlated to Cognitive Performance and White Matter Hyperintensities in Older Hypertensive Patients with Subjective Memory Complaints. Stroke, 40, 1229-1236. http://dx.doi.org/10.1161/STROKEAHA.108.532853

[10] Shenkin, S.D., Bastin, M.E., MacGillivray, T.J., et al. (2010) Carotid Intima-Media Thickness and Cerebrovascular Disease in Community-Dwelling Older People without Stroke. Stroke, 41, 2083-2086. http://dx.doi.org/10.1161/STROKEAHA.110.590505

[11] Kidwell, C.S., El-Saden, S., Livshits, Z., Martin, N.A., Glenn, T.C. and Saver, J.L. (2001) Transcranial Doppler Pulsatility Indices as a Measure of Diffuse Small-Vessel Disease. Journal of Neuroimaging, 11, 229-235. http://dx.doi.org/10.1111/j.1552-6569.2001.tb00039.x

[12] Kurata, M., Okura, T., Watanabe, S. and Higaki, J. (2005) Association between Carotid Hemodynamics and Asymptomatic White and Gray Matter Lesions in Patients with Essential Hypertension. Hypertension Research, 28, 797-803. http://dx.doi.org/10.1291/hypres.28.797

[13] Chobanian, A.V., Bakris, G.L., Black, H.R., Cushman, W.C., Green, L.A., Izzo Jr., J.L., et al. (2003) The Seventh Report of the Joint National Committee on Prevention, Detection, Evaluation, and Treatment of High Blood Pressure: The JNC 7 Report. Journal of the American Medical Association, 289, 2560-2571. http://dx.doi.org/10.1001/jama.289.19.2560

[14] Scheie, H.G. (1953) Evaluation of Ophthalmoscopic Changes of Hypertension and Arteriolar Sclerosis. AMA Archives of Ophthalmology, 49, 117-138. http://dx.doi.org/10.1001/archopht.1953.00920020122001

[15] Cockroft, D.W. and Gault, M.H. (1976) Prediction of Creatinine Clearance from Serum Creatinine. Nephron, 16, 31-41. http://dx.doi.org/10.1159/000180580

[16] González-Quevedo, A., García, S.G., Concepción, O.F., Freixas, R.S., Sotolongo, L.Q., Menéndez, M.C., et al. (2011) Increased Serum S-100B and Neuron Specific Enolase-Potential Markers of Early Nervous System Involvement in Essential Hypertension. Clinical Biochemistry, 11, 154-159. http://dx.doi.org/10.1016/j.clinbiochem.2010.11.006

[17] Henskens, L.H., van Oostenbrugge, R.J., Kroon, A.A., Hofman, P.A., Lodder, J. and de Leeuw, P.W. (2009) Detection of Silent Cerebrovascular Disease Refines Risk Stratification of Hypertensive Patients. Journal of Hypertension, 27, 846-853. http://dx.doi.org/10.1097/HJH.0b013e3283232c96

[18] Hernández-González, G., Bringas-Vega, M.L., Galán-García, L., Bosch-Bayard, J., et al. (2011) Multimodal Quantitative Neuroimaging Databases and Methods: The Cuban Brain Mapping Project. Clinical EEG and Neuroscience, 42, 1-12. http://dx.doi.org/10.1177/155005941104200303

[19] Mancia, G., Laurent, S., Agabiti-Rosei, E., Ambrosioni, E., Burnier, M., Caulfield, M.J., et al. (2009) Reappraisal of European Guidelines on Hypertension Management: A European Society of Hypertension Task Force document. Blood Pressure, 18, 308-347. http://dx.doi.org/10.3109/08037050903450468

[20] Pantoni, L. (2010) Cerebral Small Vessel Disease: From Pathogenesis and Clinical Characteristics to Therapeutic Challenges. The Lancet Neurology, 9, 689-701. http://dx.doi.org/10.1016/S1474-4422(10)70104-6

[21] Liao, D., Cooper, L., Cai, J., Toole, J.F., Bryan, N., Hutchinson, R.G., et al. (1996) Presence and Severity of Cerebral White Matter Lesions and Hypertension, Its Treatment, and Its Control. The ARIC Study. Stroke, 27, 2262-2270. http://dx.doi.org/10.1161/01.STR.27.12.2262

[22] Longstreth, W.T., Manolio, T.A., Arnold, A., Burke, G.L., Bryan, N., Jungreis, A., et al. (1996) For the Cardiovascular Health Study Collaborative Research Group. Clinical Correlates of White Matter Findings on Cranial Magnetic Resonance Imaging of 3301 Elderly People. The Cardiovascular Health Study. Stroke, 27, 1274-1282. http://dx.doi.org/10.1161/01.STR.27.8.1274

[23] Van Boxtel, M., Henskens, L., Kroon, A., Hofman, P., Gronenschild, E., Jolles, J., et al. (2006) Ambulatory Blood Pressure, Asymptomatic Cerebrovascular Damage and Cognitive Function in Essential Hypertension. Journal of Human Hypertension, 20, 5-13. http://dx.doi.org/10.1038/sj.jhh.1001934

[24] Scuteri, A., Nilssonb, P., Tzourioc, C., Redond, J. and Laurente, S. (2011) Microvascular Brain Damage with Aging and Hypertension: Pathophysiological Consideration and Clinical Implications. Journal of Hypertension, 29, 14691477. http://dx.doi.org/10.1097/HJH.0b013e328347cc17

[25] Henskens, L.H., Kroon, A.A., van Oostenbrugge, R.J., Gronenschild, E.H., Hofman, P.A., Lodder, J., et al. (2009) Associations of Ambulatory Blood Pressure Levels with White Matter Hyperintensity Volumen in Hypertensive Patients. 
Journal of Hypertension, 27, 1446-1452. http://dx.doi.org/10.1097/HJH.0b013e32832b5204

[26] Kurata, M., Okura, T., Watanabe, S. and Higaki, J. (2005) Association between Carotid Hemodynamics and Asymptomatic White and Gray Matter Lesions in Patients with Essential Hypertension. Hypertension Research, 28, 797-803. http://dx.doi.org/10.1291/hypres.28.797

[27] Heliopoulos, I., Artemis, D., Vadikolias, K., Tripsianis, G., Piperidou, C. and Tsivgoulis, G. (2012) Association of Ultrasonographic Parameters with Subclinical White-Matter Hyperintensities in Hypertensive Patients. Cardiovascular Psychiatry and Neurology, 2012, Article ID: 616572. http://dx.doi.org/10.1155/2012/616572

\section{List of Abbreviations}

WMH-white matter hyperintensities

DVRS_-Dilated Virchow-Robin spaces

LI-Lacunar infarcts

IMT_-Intima-Media thickness

PSV_Peak systolic velocity

PDV_-Peak diastolic velocities

MVF-mean velocity of flow

RI-Resistance index

PI-Pulsatility index

DD_-Diastolic dysfunction

RW-Rear wall

IVS-Interventricular septum 